\title{
A Simple Relation for the Concentration Dependence of Osmotic Pressure and Depletion Thickness in Polymer Solutions
}

\author{
Gerard J. Fleer, Alexander M. Skvortsov, Remco Tuinier*
}

We propose simple expressions $\Pi / \Pi_{0}=1+\left(\varphi / \varphi_{\mathrm{ex}}\right)^{3 \alpha-1}$ and $\left(\delta_{0} / \delta\right)^{2}=1+\left(\varphi / \varphi_{\mathrm{ex}}\right)^{2 \alpha}$ for the osmotic pressure $\Pi$ and the depletion thickness $\delta$ as a function of the polymer concentration $\varphi$. Here, $\Pi_{0}$ and $\delta_{0}$ correspond to the dilute limit, and $\varphi_{\mathrm{ex}}$ is an extrapolation concentration which is of the order of the overlap concentration $\varphi_{\text {ov }}$. The De Gennes exponent $\alpha$ describes the concentration dependence of the semidilute correlation length $\xi \sim \varphi^{-\alpha}$; it is related to the Flory exponent $v$ through $\alpha=v /(3 v-1)$. The quantity $\varphi_{\mathrm{ex}}$ is experimentally accessible by extrapolating the semidilute limit towards $\Pi=\Pi_{0}$ or $\delta=\delta_{0}$. These expressions are exact in mean field, where the ratio $\varphi_{\mathrm{ex}} / \varphi_{\mathrm{ov}}(0.49$ for $\Pi$, 0.41 for $\delta$ ) follows from established models. For excluded-volume chains they describe simulation data excellently: in this case $\varphi_{\mathrm{ex}} / \varphi_{\text {ov }}$ is 0.69 for $\Pi$ and again 0.41 for $\delta$. We find also very good agreement with experimental data.

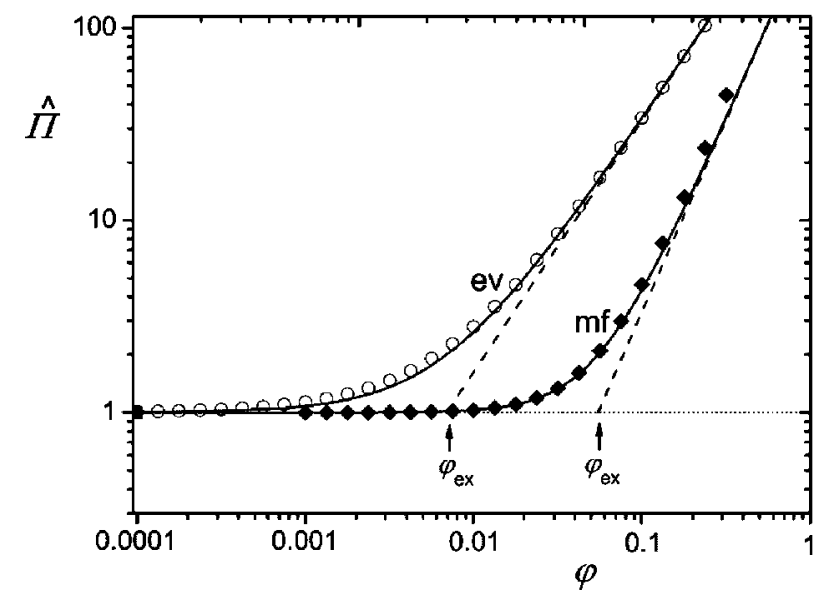

\section{Introduction}

There exists a general interest in the dependence of the osmotic pressure $\Pi$ and the depletion thickness $\delta$ in polymer solutions on the concentration $\varphi \cdot{ }^{[1,2]}$ The osmotic pressure is an important quantity for, for example, determining polymer molar masses. ${ }^{[3]}$ The depletion thickness

G. J. Fleer

Laboratory of Physical Chemistry and Colloid Science, Wageningen University, $6703 \mathrm{HB}$, The Netherlands

A. M. Skvortsov

Chemical-Pharmaceutical Academy, Prof. Popova 14, 197022 St.

Petersburg, Russia

R. Tuinier

IFF, Weiche Materie, Forschungszentrum Jülich, Institut für

Festkörperforschung, 52425 Jülich, Germany

E-mail: r.tuinier@fz-juelich.de is the size of the depletion zone (which is void of polymer) for nonadsorbing polymer next to a wall; it is the zeroth moment of the concentration profile, ${ }^{[2,4]}$ and can be measured experimentally. ${ }^{[5]}$ This characteristic length is in dilute solutions roughly equal to the radius of gyration $R$ of the polymer coils. For semidilute solutions it is essentially the correlation length $\xi$, which may be measured by, for example, scattering techniques. ${ }^{[6,7]}$ Moreover, $\Pi(\varphi)$ and $\delta(\varphi)$ play a key role in the phase behavior of colloids in the presence of nonadsorbing polymer, ${ }^{[8]}$ and $\delta(\varphi)$ is important in size-exclusion chromatography of polymers, ${ }^{[9]}$ widely used in the separation of polymers according to their chain length. ${ }^{[10]}$

Various theories have been presented to describe the equilibrium properties of moderately concentrated polymer solutions. For a theta solvent, mean-field approximations are adequate, and early versions for a good solvent were based upon a perturbation expansion in terms of the 
excluded-volume parameter; for an overview of these theories see ref. ${ }^{[3]}$. These perturbation theories cannot explain that the ratio $\Pi / \varphi$ is only a function of the normalized concentration $\varphi / \varphi_{\text {ov }}$ (where $\varphi_{\text {ov }}$ is the overlap concentration), as is found experimentally ${ }^{[3]}$.

The introduction of scaling theory (see, e.g., ref. ${ }^{[6,7]}$ ) offered an entirely new perspective. A very important step was the formulation of scaling laws for semidilute solutions in terms of $\varphi / \varphi_{\text {ov }}$ only. It should be emphasized, however, that scaling theory considers only separate regimes (in our case the dilute and semidilute regimes) and that the limiting relations are only valid for long chains. In order to describe both concentration regimes and the transition region in between, computer simulations are often used. ${ }^{[11-13]}$ They give only numerical answers which, in favorable cases, may be fitted to some pre-assumed form of an equation. An alternative is renormalization group (RG) theory, ${ }^{[14-17]}$ which in most cases leads to lengthy equations.

When a quantity of interest ( $\Pi$ or $\delta$, or some other quantity) is plotted double-logarithmically as a function of the concentration $\varphi$, generally there is a linear (chain-length dependent) behavior in the dilute limit, and another straight (chain-length independent) line in the semidilute limit, corresponding to the appropriate power law in $\varphi$. Clearly, there is a transition region between these two limits, which is situated around the overlap concentration.

It would be desirable to have a simple description for such a general behavior; this appears to be possible, as we show in this paper. We focus on the osmotic pressure and the depletion thickness, but our procedure, based upon extrapolating the semidilute behavior towards the dilute limit, can probably be generalized to other quantities as well.

In both cases we start with a scaling picture describing the two limits and propose a combination rule. Next, we show that in mean field (MF) this rule can be derived from theoretical models which are verified by numerical selfconsistent-field theory. This yields results valid for polymer chains in a theta solvent. We then generalize to chains with excluded volume (EV). We maintain the same form of the equations but replace the MF exponents (for a theta solvent) by EV exponents, and compare with renormalization-group theory and simulations. We also give some examples showing the validity of our equations to describe experimental data. Finally, we apply our model to some other quantities: osmotic compressibility and two-dimensional pressure.

\section{Osmotic Pressure}

\section{Scaling Picture}

Two Limits

In polymer solutions, there is a dilute regime below the overlap concentration $\varphi_{\mathrm{ov}}$, and a semidilute regime above it. In the dilute regime the typical length scale is the radius of gyration $R$, which depends on the chain length $N$ but not on the concentration $\varphi$; it scales as $R \sim N^{v}$, where $v$ is the Flory exponent. In the semidilute regime the length scale is the blob size (correlation length) $\xi$, which depends on $\varphi$ but not on $N$; it scales as $\xi \sim \varphi^{-\alpha}$, where the De Gennes exponent $\alpha$ is directly related to $v$ :

$$
\alpha=\frac{v}{3 v-1}, \quad(3 \alpha-1)(3 v-1)=1
$$

In MF, which is appropriate for a theta solvent, $v=$ $1 / 2$ and $\alpha=1 .{ }^{[6,7]}$ For EV chains in a good solvent the classical Flory-De Gennes result is $v=3 / 5$ and $\alpha=3 / 4 .{ }^{[1,6]}$ Renormalization group (RG) theory gives $v=0.588$ and $\alpha=0.77$. $^{\text {[7] }}$

The overlap concentration $\varphi_{\mathrm{ov}}$ separating the two regimes is defined as $\varphi_{\mathrm{ov}}=N / V_{\text {coil }}$ :

$$
\varphi_{\mathrm{ov}}=\frac{3}{4 \pi} \frac{N}{R^{3}}, \quad \varphi_{\mathrm{ov}} \sim N^{1-3 v}=N^{1 /(1-3 \alpha)}
$$

In this paper we express all length scales in units of the Kuhn length (bond length) $l$, so $R$ is dimensionless.

For the osmotic pressure, which we also express in dimensionless form (units $k T / l^{3}$ ), we have the following limits:

$$
\begin{cases}\Pi_{0}=\varphi / N & \text { dilute } \\ \Pi \sim \xi^{-3} \sim \varphi^{3 \alpha} & \text { semidilute }\end{cases}
$$

It is convenient to consider the reduced osmotic pressure $\hat{\Pi} \equiv \Pi / \Pi_{0}$. In the semidilute limit $\hat{\Pi} \sim N \varphi^{3 \alpha-1}$ which, with $N \sim \varphi_{\mathrm{ov}}^{1-3 \alpha}$ (Equation (2)), may be written in terms of $\varphi / \varphi_{\text {ov }}$, giving the following form for $\hat{\Pi}$ :

$$
\begin{cases}\hat{\Pi}=1 & \text { dilute } \\ \hat{\Pi} \sim\left(\varphi / \varphi_{\text {ov }}\right)^{3 \alpha-1} & \text { semidilute }\end{cases}
$$

Hence, in the semidilute limit $\hat{\Pi}$ is only a function of the reduced concentration $\varphi / \varphi_{\mathrm{ov}}$.

\section{Combination Rule}

So far we have used only scaling concepts, considering the proper exponents without the numerical prefactors. Moreover, we did not get any information on the transition region between the dilute and semidilute limits. Many attempts ${ }^{[3]}$ have been undertaken in the literature to describe the transition region in terms of a series expansion in concentration; such an approach is appropriate for relatively low concentrations but is not useful ${ }^{[18]}$ for higher (semidilute) concentrations. 
We propose a simple linear combination of the two limiting forms: $\hat{\Pi}=1+A\left(\varphi / \varphi_{\mathrm{ov}}\right)^{3 \alpha-1}$, where $A$ is some constant of order unity. This equation reduces to Equation (4) in both limits. When the value of the constant $A$ can be found, we might be able to describe also the transition region between the two limits.

We introduce an 'extrapolation concentration' $\varphi_{\mathrm{ex}}=A^{1 /(1-3 \alpha)} \varphi_{\mathrm{ov}}$ that, apart from a numerical constant, equals $\varphi_{\text {ov }}$ :

$$
\begin{aligned}
& \hat{\Pi}=1+\left(\varphi / \varphi_{\mathrm{ex}}\right)^{3 \alpha-1} \\
& \varphi_{\mathrm{ex}}=c \varphi_{\mathrm{ov}}
\end{aligned}
$$

where the constant $c=A^{1 /(1-3 \alpha)}=A^{1-3 v}$ is of order unity. The subscript index ex denotes extrapolation: $\varphi_{\mathrm{ex}}$ is found from extrapolating, in a double-logarithmic plot $\hat{\Pi}(\varphi)$, the semidilute straight line $\hat{\Pi}=\left(\varphi / \varphi_{\mathrm{ex}}\right)^{3 \alpha-1}$ towards $\hat{\Pi}=1$.

For MF there is an accurate description in the classical Flory-Huggins theory; we shall show below that Equation (5) (with $\alpha=1$ ) is automatically obeyed, and we shall find the numerical constant $c$ in Equation (6) $(c=0.49)$. For EV we refer to RG theory (with $\alpha=0.77$ ), and we will deduce that in that model $c=0.69$. Finally, we put Equation (5) to the test against the experimental data of Noda et al., ${ }^{[19]}$ where the (experimental) value of $v$ is 0.585 , corresponding to $3 \alpha-1=1.325$, and the best fit to the experimental data gives $c=0.8$.

\section{Osmotic Pressure in a Theta Solvent}

The Flory-Huggins (FH) expression for the osmotic pressure in a theta solvent is:

$$
\Pi=\frac{\varphi}{N}-\ln (1-\varphi)-\varphi-\frac{1}{2} \varphi^{2} \quad \Pi \approx \frac{\varphi}{N}+\frac{1}{3} \varphi^{3}
$$

From Equation (7b) we have a very simple equation for $\hat{\Pi}=\Pi / \Pi_{0}$ :

$$
\hat{\Pi}=1+\frac{N}{3} \varphi^{2}
$$

When we make a double-logarithmic plot $\hat{\Pi}(\varphi)$, we obtain Figure 1. In this example $N=1000$. The semidilute part is given by $\hat{\Pi}=(N / 3) \varphi^{2}$. The semidilute straight line with slope $2(=3 \alpha-1)$ extrapolates to $\varphi=\varphi_{\mathrm{ex}}$ at $\hat{\Pi}=1$, so $\varphi_{\mathrm{ex}}=\sqrt{3 / N}$. Therefore, Equation (8) may be written as $\hat{\Pi}=1+\left(\varphi / \varphi_{\mathrm{ex}}\right)^{2}$, which is Equation (5) with $\alpha=1$. In this case Equation (5) is identical to the (expanded) $\mathrm{FH}$ expression over the whole range of $\varphi$, including the transition region: it is not just an interpolation equation, but it follows straightforwardly from Equation (7b).

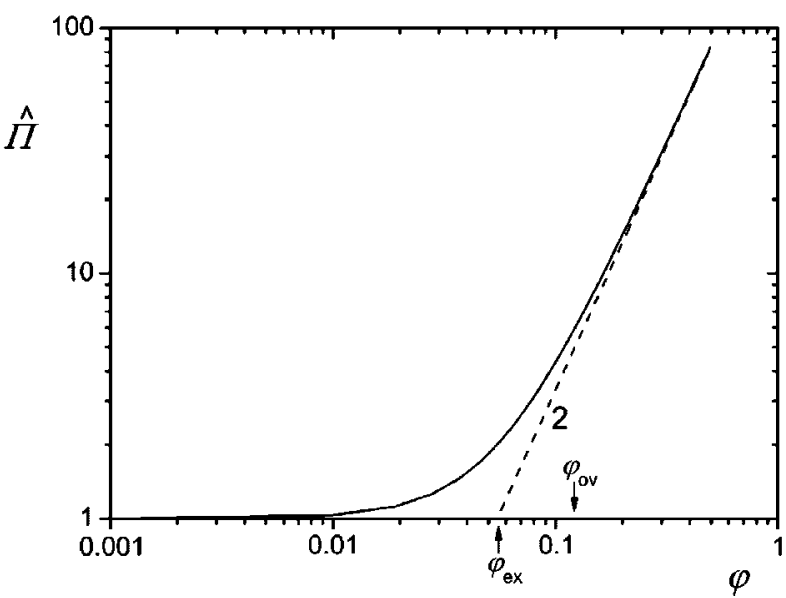

Figure 1. Double-logarithmic plot $\hat{\Pi}(\varphi)$ according to Equation (8), for $N=1000$. The semidilute line, with slope 2 , extrapolates to the extrapolation concentration $\varphi_{\mathrm{ex}}(=0.055$ in this case), the overlap concentration $\varphi_{\mathrm{ov}}$ is 0.11 ; these two concentrations are indicated by arrows.

The next point is the relation between $\varphi_{\mathrm{ex}}$ and $\varphi_{\mathrm{ov}}$. Since $\varphi_{\mathrm{ex}}$ in Figure 1 is in the transition region, we expect that $\varphi_{\mathrm{ex}}$ is of order $\varphi_{\mathrm{ov}}$. Indeed, according to Equation 2 with $R=\sqrt{N / 6}$ we have $\varphi_{\text {ov }}=3.51 N^{-1 / 2}$. Hence, in Equation (6) for a theta solvent $c=\sqrt{3} / 3.51$ :

$$
\hat{\Pi}=1+\left(\varphi / \varphi_{\mathrm{ex}}\right)^{2} \quad \varphi_{\mathrm{ex}}=0.49 \varphi_{\mathrm{ov}}
$$

With this expression for $\hat{\Pi}=\Pi / \Pi_{0}$ we have a simple equation for theta conditions that is valid over the entire concentration range, and is nothing else than a reformulation of the (expanded) Flory-Huggins expression.

\section{Osmotic Pressure for Excluded-Volume Chains}

For EV chains we can rely on computer simulations ${ }^{[11-13]}$ or on renormalization group (RG) theory. ${ }^{[14-17]}$ For describing the osmotic pressure we choose for the RG approach because there are some analytical results available. In RG theory the properties of polymer solutions (e.g., osmotic pressure, or chain dimensions) are obtained in the form of a series expansion in a parameter $\varepsilon=4-d$, where $d$ is the space dimensionality and $\varepsilon$ is assumed to be small. Usually, only first-order results can be obtained in an analytical form. For three-dimensional space $(d=3)$ the parameter $\varepsilon$ is set to unity.

Ohta and Oono ${ }^{[15]}$ derived the following RG expression for the reduced osmotic pressure $\hat{\Pi}=\Pi / \Pi_{0}$ :

$$
\begin{aligned}
\hat{\Pi} & =1+\frac{\chi}{2} e^{\left[1 / x+\left(1-1 / x^{2}\right) \ln (1+x)\right] / 4} \\
\chi & =B \varphi / \varphi_{\text {ov }}
\end{aligned}
$$


where $B$ is an unknown constant of order unity. For large $x$ this equation gives $\hat{\Pi} \sim \varphi^{1+\varepsilon / 4}=\varphi^{1.25}$, a slightly weaker dependence than $\hat{\Pi} \sim \varphi^{3 \alpha-1}=\varphi^{1.309}$ which is in agreement with experiment. Various attempts ${ }^{[16,17]}$ have been made to improve on this exponent, resulting in even more complicated expressions.

Schaefer ${ }^{[18]}$ gives an RG expression for $\hat{\Pi}$ which avoids the unknown constant $B$ :

$$
\begin{aligned}
\hat{\Pi}= & 1+1.314 \bar{\varphi}\left\{\frac{1+2.4167 \bar{\varphi}+0.9956 \bar{\varphi}^{2}}{1+0.5099 \bar{\varphi}}\right\}^{3 \alpha-2} \\
& 3 \alpha-2=0.309
\end{aligned}
$$

where $\bar{\varphi}=\varphi / \varphi_{\mathrm{ov}}$ and $\alpha=0.77$. In the semidilute limit $\bar{\varphi} \gg 1$ :

$$
\hat{\Pi}=1.616\left(\varphi / \varphi_{\text {ov }}\right)^{3 \alpha-1} \quad 3 \alpha-1=1.309
$$

The same limit from Equation (5) is $\hat{\Pi}=\left(\varphi / \varphi_{\mathrm{ex}}\right)^{3 \alpha-1}$, so when we identify the two limits we find $c=\varphi_{\mathrm{ex}} / \varphi_{\mathrm{ov}}=1.616^{-1 / 1.309}=0.69$. Hence, with Equation 5:

$$
\hat{\Pi}=1+\left(\varphi / \varphi_{\mathrm{ex}}\right)^{1.309} \varphi_{\mathrm{ex}}=0.69 \varphi_{\mathrm{ov}}
$$

We propose Equation (13) as a simple alternative to Equation (11). Figure 2 (left part labeled ev) shows the comparison. The circles in Figure 2 represent Equation (11), and the solid curve is Equation (13). Arbitrarily, we chose $\varphi_{\mathrm{ov}}=0.01$ (which fixes the chain length because $\varphi_{\mathrm{ov}} \sim N^{1 /(1-3 \alpha)}$, see Equation (2)). The agreement is

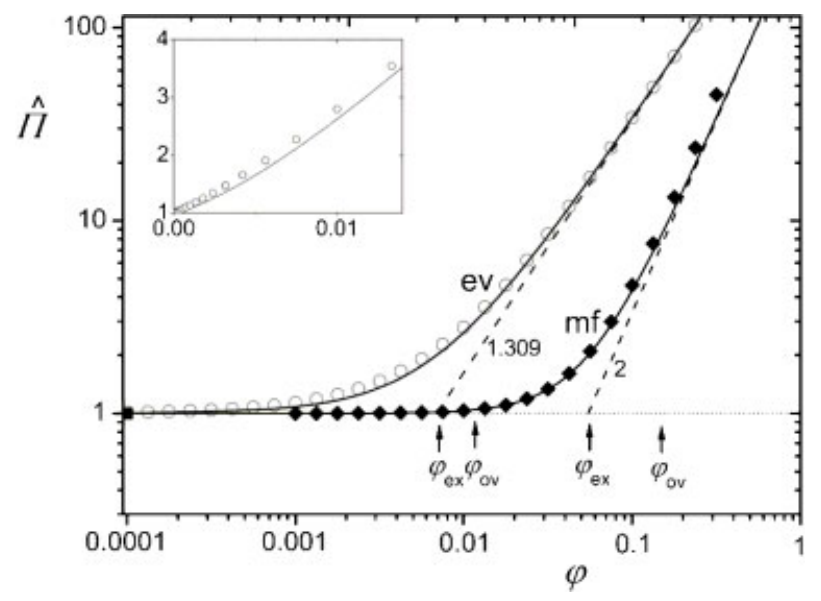

Figure 2. Comparison of Equation (11) (circles) with Equation (13) for EV chains (left part labeled EV). The right part labeled MF is the same as in Figure 1; the diamonds follow the full $\mathrm{FH}$ expression (Equation (7a)). The slope of the semidilute straight lines (dashed) is indicated. The values of $\varphi_{\mathrm{ex}}$ and $\varphi_{\mathrm{ov}}$ are shown by arrows. The inset shows more detail of the dilute behavior for $\mathrm{EV}$, on a linear scale. excellent, over the entire concentration region, so our simple equation works quite well. We see again that, given the RG data points, the value of $\varphi_{\text {ex }}$ is easily obtained by extrapolating the semidilute straight line towards $\hat{\Pi}=1$.

We note that Equation (13) is mathematically not exact in the dilute regime, which is the relevant region for determining the second virial coefficient. The inset of Figure 2 gives the dilute range on a linear scale. The symbols are again Equation (11), and the solid curve is Equation (13). The RG result corresponds to a linear concentration dependence $\hat{\Pi}=1+1.314 \varphi / \varphi_{\mathrm{ov}}$ for low $\varphi$, whereas in Equation (13) the initial part is not straight. Nevertheless, even in the dilute region Equation (13) is a reasonable approximation.

For comparison, Figure 2 shows also the MF data of Figure 1, but we added the full $\mathrm{FH}$ expression (Equation (7a), diamonds). We see the expected deviations with the expanded form (Equation (7b), solid curve) for $\varphi>0.3$, caused by higher-order terms in the expansion of the logarithm.

\section{Comparison with Experimental Data}

Figure 3 shows experimental data by Noda et al., ${ }^{[19]}$ for narrow fractions of poly $(\alpha$-methylstyrene) of five molar masses, in the form $\hat{\Pi}$ against $\varphi / \varphi_{\mathrm{ov}}$. We compare these data to two RG equations, Equation (10) (dashed curve) and Equation (11) (dotted), and to Equation (5) (solid curve). In Equation (10) we used $B=1$, and we see that then Equation (10) seriously underestimates the experimental data. The fit could be improved considerably by choosing,

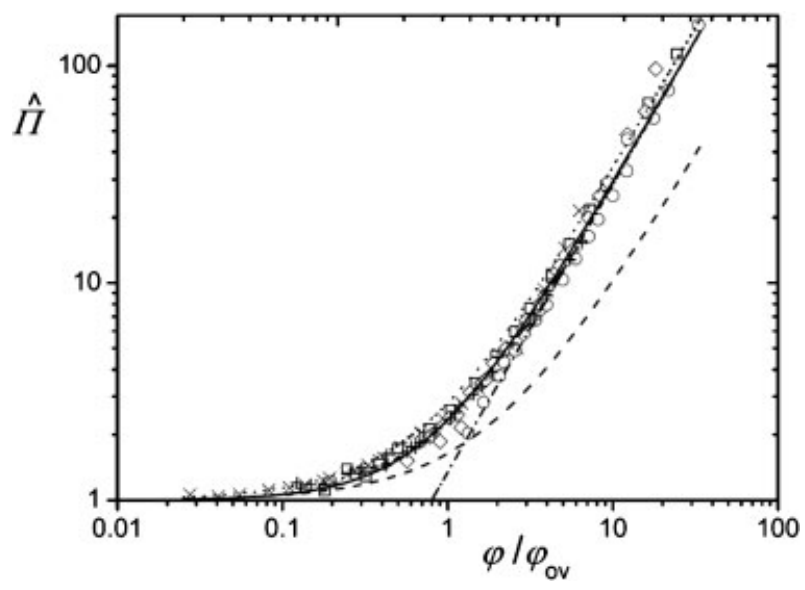

Figure 3. Comparison of the experimental data of Noda et al. ${ }^{[19]}$ with Equation (5), (10) and (11). The plot gives $\hat{\Pi}\left(\varphi / \varphi_{\text {ov }}\right)$ on a double-logarithmic scale. Crosses, plusses, squares, diamonds, and circles are the experimental data for $70,200,506,1190$, and $1820 \mathrm{~kg} \cdot \mathrm{mol}^{-1}$, respectively. The solid curve is Equation (5), the dashed curve is Equation (10) with $B=1$, and the dotted curve is Equation (11). The semidilute straight line (dot-dashed) is $\hat{\Pi}=\left(\varphi / \varphi_{\mathrm{ex}}\right)^{1.325}$, with $\varphi_{\mathrm{ex}}=0.8 \varphi_{\mathrm{ov}}$. 
for example, $B=2.5$ (not shown). Equation (11) (which implies $3 \alpha-1=1.309$ for the semidilute slope) works much better: it describes the two limits quite well, and gives a slight overestimation in the transition region.

Next we turn to Equation (5), based upon our extrapolation procedure from the semidilute region as described before. Noda et al. reported an experimental value $v=0.585$, corresponding to $3 \alpha-1=1.325$. We use this slope 1.325 for the semidilute straight line, and shift this line horizontally to find the best fit with the semidilute data points; we find $\varphi_{\mathrm{ex}}=0.80 \varphi_{\mathrm{ov}}$. The numerical constant $c$ is close to the RG result 0.69 in Equation (13). When we use this $\varphi_{\text {ex }}$ in Equation (5) with $3 \alpha-1=1.325$, we find the solid curve in Figure 3 . The fit is very good: the performance of our simple equation is gratifying.

\section{Depletion Thickness}

\section{Scaling Picture}

\section{Two Limits}

In a scaling picture the depletion thickness $\delta$ has the following limits:

$$
\begin{cases}\delta_{0} \approx R \sim N^{v} \sim \varphi_{\mathrm{ov}}^{-\alpha} & \text { dilute } \\ \delta \approx \xi \sim \varphi^{-\alpha} & \text { semidilute }\end{cases}
$$

The last proportionality in Equation (14a), $N^{v} \sim \varphi_{\mathrm{ov}}^{-\alpha}$, follows from $N \sim \varphi_{\text {ov }}^{1-3 \alpha}$ (Equation (2)) in combination with $v=\alpha /(3 \alpha-1)$ (Equation (1)). We see that in the semidilute regime the reduced depletion thickness $\hat{\delta}=\delta / \delta_{0}$ follows the scaling law $\hat{\delta} \sim\left(\varphi / \varphi_{\text {ov }}\right)^{-\alpha}$.

\section{Combination Rule}

For the osmotic pressure (Equation (5)) we used a linear combination of $\hat{\Pi}$ for the two limits. For the depletion thickness, a linear combination of the type $\hat{\delta}=1+A\left(\varphi / \varphi_{\text {ov }}\right)^{-\alpha}$ does not work, as the limit for $\varphi \rightarrow 0$ would diverge. Instead, we make a linear combination of $\hat{\delta}^{-2}$. This is based upon the results of an earlier paper, ${ }^{[4]}$ where we have shown that the inverse square of $\delta$ is equal to the sum of the inverse squares of $\delta_{0}$ and $\xi$ :

$$
\delta^{-2}=\delta_{0}^{-2}+\xi^{-2}
$$

In that paper we used mean-field expressions for $\delta_{0}$ and $\xi$, for both a theta solvent (where MF is correct) and good solvents (where the MF exponents are wrong). In this paper we shall substitute the correct exponents for EV, but we assume that the form of Equation (15) remains valid.
We then need $\hat{\delta}^{-2}$ :

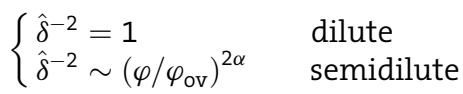

A similar linear combination of the two limits as for the osmotic pressure (see Equation (5)) then leads to the Ansatz:

$$
\hat{\delta}^{-2}=1+\left(\varphi / \varphi_{\mathrm{ex}}\right)^{2 \alpha}
$$

where $\varphi_{\mathrm{ex}}=c \varphi_{\mathrm{ov}}$ is again an extrapolation concentration which is of order $\varphi_{\text {ov }}$. The numerical constant $c$ for the depletion thickness might be different, however, from that for the osmotic pressure.

In the next section we will show that Equation (17) for a theta solvent $(2 \alpha=2)$ follows automatically from our earlier result (Equation (15)); the constant $c$ is 0.41 . For EV (see below), we will make a comparison with simulation results by Louis et al.; ${ }^{[20,21]}$ we will see that Equation (17) works well with $\alpha=0.77$ and - surprisingly - the same constant $c=0.41$. We also consider some other simulation data. Finally, we use experimental data to test Equation (17).

\section{Depletion Thickness in a Theta Solvent}

We use Equation (15) with MF expressions for $\delta_{0}$ and $\xi$. The dilute limit is well known: $:^{[4,22,23]}$

$$
\delta_{0}=\frac{2}{\sqrt{\pi}} R=2 \sqrt{\frac{N}{6 \pi}}, \quad \delta_{0}^{-2}=\frac{3 \pi}{2 N}
$$

For the semidilute limit in a theta solvent we derived before: ${ }^{[4]}$

$$
\xi^{-2}=-\frac{3}{p^{2}}[\ln (1-\varphi)+\varphi] \xi^{-2} \approx \frac{3}{2 p^{2}} \varphi^{2}
$$

The constant $p$ equals $\sqrt{3 / 2} \operatorname{atanh}(1 / \sqrt{3})=0.807$. Equation (19b) shows that the De Gennes exponent in $\xi \sim \varphi^{-\alpha}$ is $\alpha=1$, as expected.

From Equation (15) we find $\hat{\delta}^{-2}=1+\delta_{0}^{2} / \xi^{2}$. Substituting Equations (18) and (19b) leads to:

$$
\hat{\delta}^{-2}=1+\frac{N}{\pi p^{2}} \varphi^{2}
$$

Figure 4 (right part labeled MF) gives a doublelogarithmic plot $\hat{\delta}(\varphi)$ according to Equation (20), for $N=1000\left(\delta_{0}=14.57\right)$. The semidilute part of this curve is given by $\hat{\delta}=p \sqrt{\pi / N} \varphi^{-1}$; its slope is $-1=-\alpha$. The semidilute straight line extrapolates to $\varphi=\varphi_{\mathrm{ex}}$, given by 


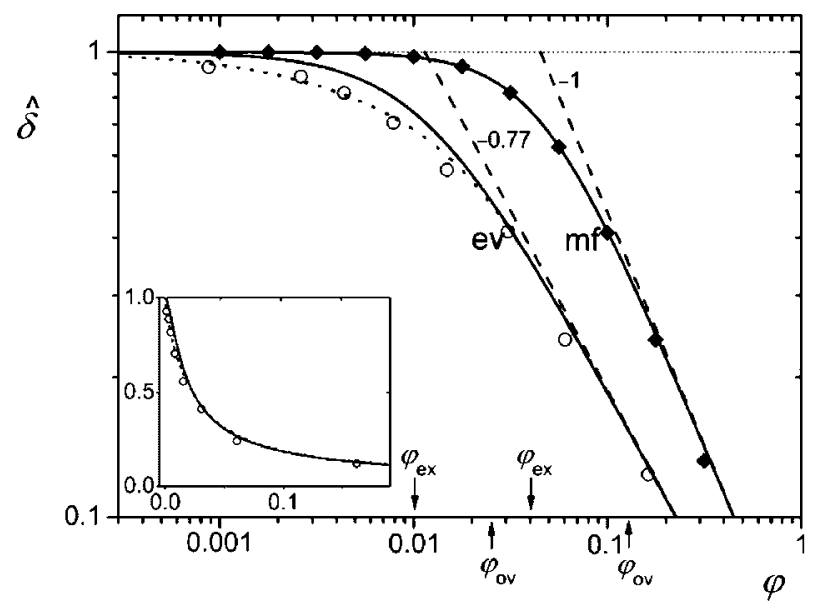

Figure 4. Comparison of our analytical depletion thickness with numerical results, for MF (right) and EV (left). Diamonds are numerical SCF results for $N=1000$ under theta conditions, the solid MF curve is Equation (21). Circles are EV simulation results by Louis et al. ${ }^{[21]}$ for $N=500$ (redrawn), the solid EV curve is Equation (23). The dotted curve (EV) is Equation (24). The slope of the semidilute straight lines (dashed) is indicated. The values of $\varphi_{\mathrm{ex}}$ and $\varphi_{\text {ov }}$ are shown by arrows. The inset gives $\delta(\varphi)$ for $\mathrm{EV}$ on a linear scale.

$\varphi_{\mathrm{ex}}=p \sqrt{\pi / N}=1.44 N^{-1 / 2}$. Therefore, Equation (20) may also be written as $\hat{\delta}^{-2}=1+\left(\varphi / \varphi_{\mathrm{ex}}\right)^{2 \alpha}$ (with $\alpha=1$ ), which is identical to Equation (17), and we see that Equation (17) is exact in mean field.

The diamonds in Figure 4 are numerical SCF results. They coincide with Equation (20) (or Equation (17) or (21) below) over most of the concentration region. Only for high concentrations are some slight deviations visible. In this region the full logarithmic form of $\xi$ (Equation (19a)), in combination with Equation (15), gives nearly quantitative agreement with numerical SCF.

The relation between $\varphi_{\mathrm{ex}}\left(=1.44 N^{-1 / 2}\right)$ and $\varphi_{\text {ov }}\left(=3.51 N^{-1 / 2}\right)$ is given by $c=1.44 / 3.51=0.41$. Therefore, the depletion thickness in a theta solvent can be written as:

$$
\hat{\delta}=\left[1+\left(\varphi / \varphi_{\mathrm{ex}}\right)^{2}\right]^{-0.5}, \quad \varphi_{\mathrm{ex}}=0.41 \varphi_{\mathrm{ov}}
$$

Again $c$ is of order unity, but for the depletion thickness the constant $c$ is somewhat smaller than for the osmotic pressure.

\section{Depletion Thickness for Excluded-Volume Chains}

Also for the depletion thickness of EV chains we would have, in principle, the choice of using computer simulations or RG theory. However, we are not aware of RG results except for the dilute case, ${ }^{[24]}$ so beyond the dilute limit we have to rely on simulations. Fortunately, some very accurate fits for the numerical simulations are available.
Basically, there are two ways to extract $\delta$ from simulation data. The first is to determine the zeroth moment of the concentration profile, finding the characteristic width of the depletion zone by fitting on some theoretical form of the profile. Louis et al. ${ }^{[21]}$ used a standard profile $\varphi(\mathrm{z}) / \varphi_{\mathrm{b}}=\tanh ^{2}(z / \delta)$, Shih et al. ${ }^{[25]}$ assumed a form $\varphi(\mathrm{z}) / \varphi_{\mathrm{b}}=1-\exp \left[-(z / \Delta)^{m}\right]$ with $m=1.63$. When a plot is made of both profiles, they basically coincide provided the conversion $\delta=1.1 \Delta$ is made; therefore both procedures give essentially the same information.

A completely different procedure is to consider polymer in a slit in equilibrium with a bulk solution, and to find the distribution coefficient $K=\varphi_{\text {in }} / \varphi_{\mathrm{b}}$, where $\varphi_{\text {in }}$ is the average concentration inside the slit. When the slit is wide enough for the depletion zones not to overlap, which is the case when $K>0.5$, the depletion thickness may be calculated from $K=1-2 \delta / D$, where $D$ is the slit width. That is how, in a previous paper, ${ }^{[26]}$ we obtained $\delta(\varphi)$ from distribution coefficients reported by Cifra et al. ${ }^{[27]}$ We showed that for wide slits $\delta(\varphi)$ does not depend on $D$.

We start with the simulation data by Louis et al. ${ }^{[21]}$ because they also provided a very accurate fit for the dependence $\delta(\varphi)$. In the dilute limit they found $\delta_{0}=1.074 R$, which is very close to the RG result by Hanke et al. ${ }^{[24]}$ who arrived at $\delta_{0}=1.071 R$ for EV chains. We write the Louis fit as:

$$
\hat{\delta}=\left[1+7.63 \varphi / \varphi_{\text {ov }}+14.56\left(\varphi / \varphi_{\text {ov }}\right)^{3}\right]^{-0.2565}
$$

For our extrapolation procedure we need only the semidilute limit, where Equation (22) reduces to:

$$
\hat{\delta}=0.503\left(\varphi / \varphi_{\text {ov }}\right)^{-0.77}
$$

In our model $\hat{\delta}=\left(\varphi / \varphi_{\mathrm{ex}}\right)^{-\alpha}$ in the semidilute limit. Hence, we take $\alpha=0.77$ and calculate the constant $c$ from $c=\varphi_{\mathrm{ex}} / \varphi_{\mathrm{ov}}=0.503^{1 / \alpha}=0.41$. Surprisingly, this is the same result as in Equation (21) for MF chains. At the moment, we do not know whether this is accidental or whether some deeper underlying concept causes this equality of the MF and EV constants.

Equation (17) gives now the form which should apply to EV chains:

$$
\hat{\delta}=\left[1+\left(\varphi / \varphi_{\mathrm{ex}}\right)^{1.54}\right]^{-0.5} \varphi_{\mathrm{ex}}=0.41 \varphi_{\mathrm{ov}}
$$

Figure 4 (left part labeled EV) gives the simulation data by Louis et al. (circles) and the comparison with Equation (24) (solid curve). In the simulations $N=500, R=16.83$, and $\varphi_{\text {ov }}=0.025{ }^{\left[{ }^{[28]}\right.}$ Hence, in Equation (24) we use $\varphi_{\text {ex }}=0.41$ $\varphi_{\mathrm{ov}}=0.0103$. The agreement is quite satisfactory, although Equation (24) overestimates the simulation data slightly in the dilute region. When plotted on a linear scale (inset 
of Figure 4) these deviations do not seem to be very serious, and Equation (24) is a convenient, yet rather accurate, approximation. The validity of our extrapolation procedure is again corroborated in Figure 4, not only for MF (where it gives an exact description), but also for EV.

The dotted curve in Figure 4 (and in the inset) is the Louis fit of Equation (22). It gives a very accurate description of the simulation results, especially in dilute solutions where the linear term in Equation (22) contributes.

In Figure 5 we compare our simple expression to simulation results of Shih et al. ${ }^{[25]}$ In this case we did not plot the normalized $\hat{\delta}$ but $\delta$ itself. We multiplied the $\Delta$ values reported by Shih et al. by 1.1 to obtain $\delta$. The simulations are for $N=100\left(R=6.3, \varphi_{\text {ov }}=0.095\right)$ and for $N=40\left(R=3.6, \varphi_{\mathrm{ov}}=0.20\right)$. The solid curves in Figure 5 represent Equation (24) with an appropriate choice for $\delta_{0}$ : we took $\delta_{0}=6.9$ for $N=100$, and $\delta_{0}=6.9(40 / 100)^{0.6}=4.0$ for $N=40$. These values are very close to $1.071 R$, the theoretical result ${ }^{[24]}$ for EV chains.

It is seen that Equation (24) describes the data for $N=40$ quite well, which shows again the validity of our extrapolation procedure. For $N=100$ the agreement is less, especially around $\varphi_{\text {ex }}$. At the moment we do not have a clear explanation for this deviation. We note that Equation (22) is even worse in this case, as this gives lower values than Equation (24) (see Figure 4).

Figure 6 gives a normalized representation $\hat{\delta}$ against $\varphi / \varphi_{\text {ov }}$, with data from three sources:

Louis et al. ${ }^{[21]}$ for $N=500$ (squares), Shih et al. ${ }^{[25]}$ for $N=100$ (crosses) and 40 (diamonds), and Cifra et al. ${ }^{[27]}$ for $N=100$ (circles). For the former two sets $R$ and $\varphi_{\text {ov }}$ are given above, for the Cifra data $R=6.45, \varphi_{\text {ov }}=0.089$ (compare $R=6.3, \varphi_{\mathrm{ov}}=0.095$ for $N=100$ in the Shih data). The distribution coefficient $K$ reported by Cifra et al. ${ }^{[27]}$ was converted to $\delta$ as described in ref. [26], using

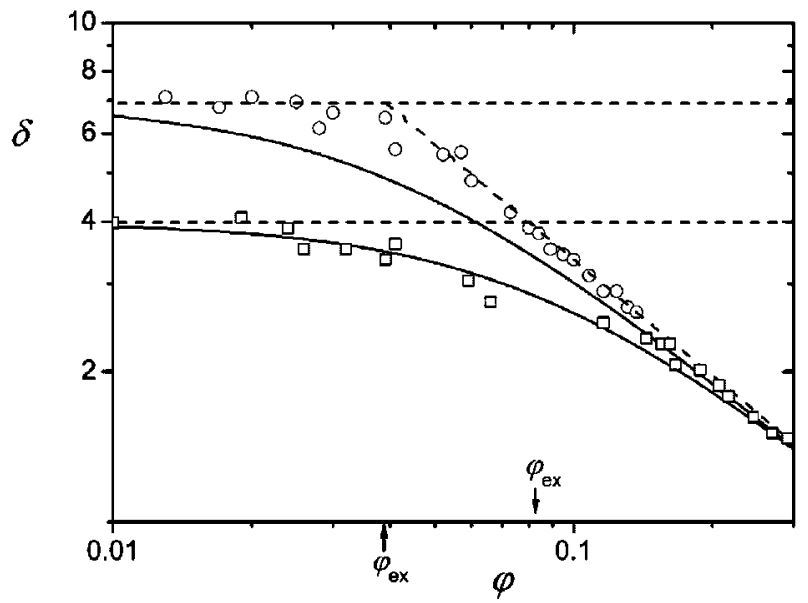

Figure 5. Simulation data $\delta(\varphi)$ by Shih et al. ${ }^{[25]}$ for $N=100$ (circles) and $N=40$ (squares), as compared to Equation (24) (solid curves). The dilute and semidilute limits are given by the dashed line.

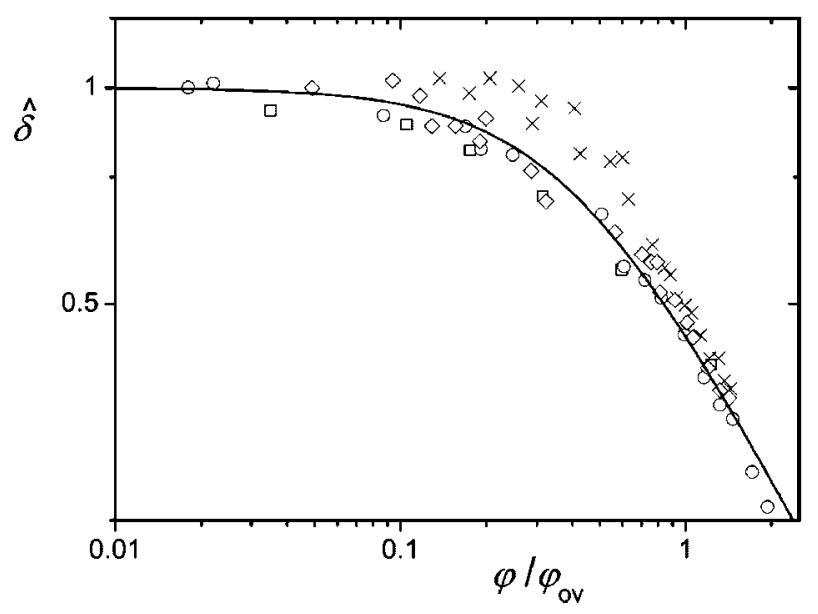

Figure 6. Normalized depletion data $\hat{\delta}\left(\varphi / \varphi_{\text {ov }}\right)$ from Louis et al. ${ }^{[21]}$ for $N=500$ (squares; redrawn), Shih et al. ${ }^{[25]}$ for $N=100$ (crosses) and 40 (diamonds), and Cifra et al. ${ }^{[27]}$ for $N=100$ (circles). The solid curve is Equation (24).

$\delta=(D / 2)(1-K)$ for wide pores $(K>0.5)$. The data points (circles) in Figure 6 are for six slit widths $D=5,7,11,19,29$, and 45; they all fall onto one curve. The solid curve in Figure 6 is Equation (24).

The overall pattern for the different simulations is the same. The Shih data for $N=100$ (crosses) fall slightly out of the pattern, with rather high values around $\varphi=\varphi_{\mathrm{ex}}$. The Louis data (squares) deviate slightly downwards in the dilute regime. The Cifra data (circles) practically coincide (over the range shown) with Equation (24). This is in line with our description of these data in ref. ${ }^{[26]}$ (where we used a slightly more complicated form of Equation (24), replacing $\varphi$ by $-\ln (1-\varphi)$ to be able to describe also high concentrations).

All in all, we conclude that our combination rule describes the simulation data quite well, not only in the dilute and semidilute limits, but also in the transition region. For very concentrated solutions (beyond the range shown in Figure 6) we would need logarithmic corrections. ${ }^{[4,26]}$

\section{Comparison with Experimental Data}

In Figure 7 we show experimental data by Satterfield et al., ${ }^{[29]}$ for nearly monodisperse fractions of polystyrene in the good solvent chloroform, in the form $\hat{\delta}$ against $\varphi / \varphi_{\text {ov }}$. These authors measured the distribution coefficient $K$ in equilibrium partitioning experiments for five molar masses (see legend), using borosilicate glass with average pore diameter $D=91.3 \mathrm{~nm}$. In this case the wide-pore regime applies, as the highest $R$ (for $670 \mathrm{~kg} \cdot \mathrm{mol}^{-1}$ ) was $36.4 \mathrm{~nm}$. We converted $K$ to $\delta$ (in nm) using $\delta=(D / 2)(1-K)$. For obtaining $\delta_{0}$ we assumed $\delta_{0}=1.071 R$, with $R$ (in $\mathrm{nm}$ ) given by $0.015 M^{0.581},{ }^{29]}$ with $M$ the relative molar mass 


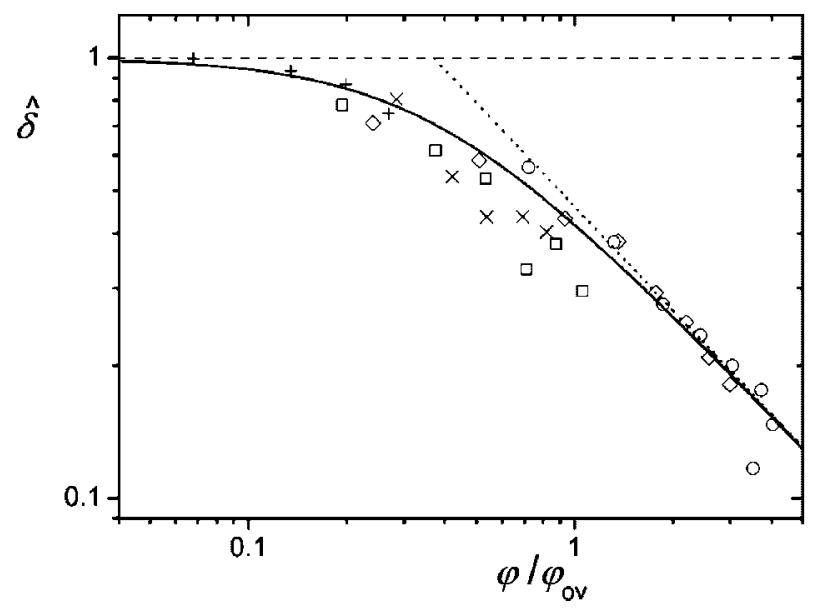

Figure 7. Comparison of the experimental data of Satterfield et al. ${ }^{[29]}$ with Equation (17). The plot gives $\hat{\delta}\left(\varphi / \varphi_{\text {ov }}\right)$ on a doublelogarithmic scale. Plusses, crosses, squares, diamonds, and circles are for $37,110,200,498$, and $670 \mathrm{~kg} \cdot \mathrm{mol}^{-1}$, respectively. The semidilute straight line (dotted) is $\hat{\delta}=\left(\varphi / \varphi_{\mathrm{ex}}\right)^{-0.782}$, with $\varphi_{\mathrm{ex}}=0.37 \varphi_{\mathrm{ov}}$. The solid curve is Equation (17).

with respect to hydrogen. The overlap concentration (mass per unit volume) was calculated from $(3 / 4 \pi) M / R^{3}$. We selected only those data for which $K<0.9$ (otherwise the experimental error for determining $\delta$ is too big), and we discarded a few data points which would lead to $\delta>\delta_{0}$.

With the experimental $v=0.581$ we find from Equation (1) $\alpha=0.782$. Hence, in Equation 17 we should use $2 \alpha=1.564$ and the slope of the semidilute line in Figure 7 should be -0.782 . Like in Figure 3 for the osmotic pressure, we shift this line horizontally to find the best fit with the semidilute data points; the result is $\varphi_{\mathrm{ex}}=0.37 \varphi_{\mathrm{ov}}$. The constant is quite close to the theoretical 0.41 in Equation (21) and (24).

The solid curve in Figure 7 is Equation (17) with these values of $\alpha$ and $\varphi_{\mathrm{ex}}$. Despite some scatter in the experimental data, we may conclude that our simple equation works quite well.

\section{Extension to Other Quantities}

\section{Osmotic Compressibility}

As we have a simple expression for $\Pi=\Pi_{0} \hat{\Pi}=(\varphi / N) \hat{\Pi}$, with $\hat{\Pi}$ given by Equation (5), it is straightforward to find the inverse osmotic compressibility $\partial \Pi / \partial \varphi$ :

$$
N \partial \Pi / \partial \varphi=1+3 \alpha\left(\varphi / \varphi_{\mathrm{ex}}\right)^{3 \alpha-1}
$$

where $\varphi_{\mathrm{ex}}=c \varphi_{\mathrm{ov}}$ is the extrapolation concentration for the osmotic pressure. We note that we could absorb the prefactor $3 \alpha$ into $\varphi_{\mathrm{ex}}$ and write $N \partial \Pi / \partial \varphi=1+\left(\varphi / \varphi_{\mathrm{ex}}^{\prime}\right)^{3 \alpha-1}$, with $\varphi_{\mathrm{ex}}^{\prime}=c^{\prime} \varphi_{\mathrm{ov}}$ the extrapolation concentration for the osmotic compressibility; clearly $c^{\prime} / c=(3 \alpha)^{1 /(1-3 \alpha)}=$ $(3 \alpha)^{1-3 v}$. This form shows that $\varphi_{\mathrm{ex}}^{\prime}$ can be found by extrapolating, in a double-logarithmic plot $N \partial \Pi / \partial \varphi$ versus $\varphi$, the semidilute straight line towards $N \partial \Pi / \partial \varphi=1$. We stick to the form of Equation (25) in order to maintain the connection with the osmotic pressure.

The compressibility can be measured experimentally from light scattering. The scattering intensity $S(q)$ per monomer unit at scattering wave vector $q$ is given by: ${ }^{[6]}$

$$
S(q)=\frac{g}{1+q^{2} \xi^{2}} \quad g=\frac{1}{N \partial \Pi / \partial \varphi}
$$

which defines the correlation length $\xi$ measured in scattering experiments. The quantity $\mathrm{Ng}$ can be regarded as the number of monomer units within one blob. ${ }^{[6]}$

From the experimental data of Noda et al. ${ }^{[19]}$ (Figure 3), which we interpreted using Equation (5) with $3 \alpha=2.325$ and $\varphi_{\mathrm{ex}}=0.8 \varphi_{\mathrm{ov}}$, we can predict the osmotic compressibility of poly ( $\alpha$-methylstyrene) in toluene with Equation (25) without any further parameters. Unfortunately, we cannot check this prediction as we are not aware of experimental compressibility data for this system.

Experimental data for $g$ as a function of $\varphi / \varphi_{\text {ov }}$ for polystyrene in toluene were obtained by at least two groups: Wiltzius et al. ${ }^{[30]}$ and Hamada et al. ${ }^{[31]}$ Wiltzius et al. compared their data with the differentiated form of Equation (10):

$$
\begin{gathered}
N \frac{\partial \Pi}{\partial \varphi}=1+\frac{1}{8}\left(9 x-2+\frac{2 \ln (1+x)}{\chi}\right) e^{\left[1 / x+\left(1-1 / x^{2}\right) \ln (1+x)\right] / 4} \\
x=\varphi / \varphi_{\mathrm{ov}}
\end{gathered}
$$

They found that Equation (27) seriously underestimates the experimental data, in the same way as Equation (10) with $B=1$ underestimates the osmotic pressure. We note that, like in Figure 3, the agreement would become much better if in Equation (27) $x=B \varphi / \varphi_{\text {ov }}$ with $B=2.5$ would be taken. Our Equation (25) would probably work as well: we cannot make a quantitative comparison as we do not have the numerical data.

We can make such a comparison with the Hamada data, as these authors tabulated their results. Figure 8 gives these data for six molar masses as given in the legend. The dotted curve is Equation (27); we see again the underestimation. For comparing with Equation (25) we need the constants $\alpha$ and $c=\varphi_{\mathrm{ex}} / \varphi_{\mathrm{ov}}$. Hamada et al. report an experimental Flory exponent $v=0.588$, which according to Equation (1) corresponds to $3 \alpha=2.309$. As these numbers are the same as those obtained from RG theory, it is reasonable to take the $c$-value from Equation (13): $c=0.69$. The solid curve in Figure 8 gives our prediction from Equation (25). The agreement is quite satisfactory. It would 


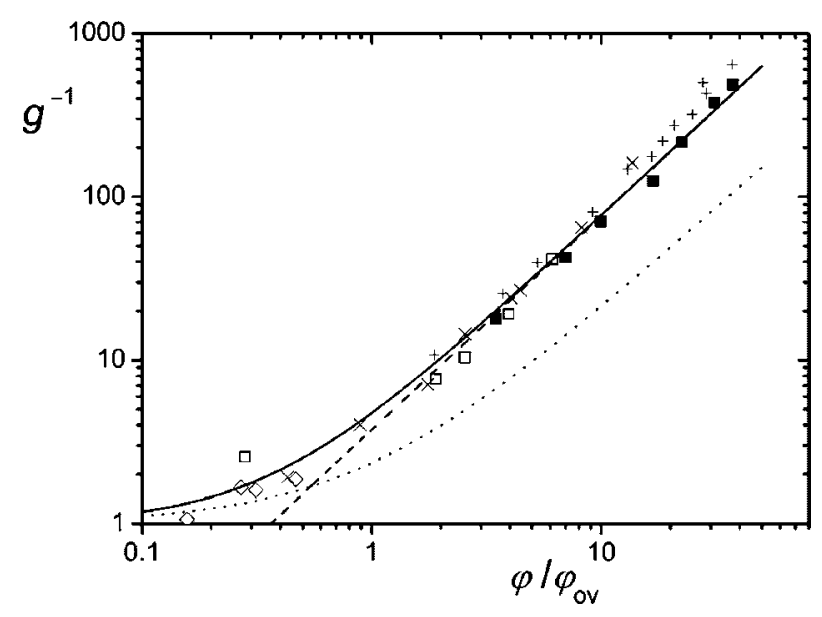

Figure 8. Comparison of the experimental data of Hamada et al. ${ }^{[31]}$ with Equation (25) and (27). The plot gives $g^{-1}=N 2 \Pi$ / $\partial \varphi$ as a function of $\varphi / \varphi_{\text {ov }}$ on a double-logarithmic scale. Filled squares, diamonds, circles, open squares, crosses, and plusses are for $17.5,37,50,110,900$, and $2000 \mathrm{~kg} \cdot \mathrm{mol}^{-1}$, respectively. The solid curve is Equation (25), the dotted curve is Equation (27). The semidilute straight line (dashed) is $g^{-1}=3 \alpha\left(\varphi / \varphi_{\mathrm{ex}}\right)^{3 \alpha-1}$, with $3 \alpha=$ 2.309 and $\varphi_{\mathrm{ex}}=0.69 \varphi_{\mathrm{ov}}$.

be possible to improve the fit somewhat by adjusting the two numerical constants slightly; we will not do that as we do not want to introduce extra parameters.

\section{Two-Dimensional Pressure}

Above, we considered the osmotic pressure of polymer chains in three-dimensional space $(d=3)$. In this final section we give an example for two-dimensional chains $(d=2)$. In $d$-dimensional space Equation (1) is generalized to:

$$
(d \alpha-1)(d v-1)=1
$$

For $d=2$ the two-dimensional radius of gyration $R$ scales as $R \sim N^{3 / 4}$, ${ }^{[7]}$ so $\nu=3 / 4$ and $\alpha=3 / 2$. The overlap concentration is defined as:

$$
\varphi_{\mathrm{ov}}=\frac{N}{\pi R^{2}} \varphi_{\mathrm{ov}} \sim N^{-1 / 2}
$$

For the two-dimensional pressure we have $\Pi \sim \xi^{-2} \sim \varphi^{3}$ in the semidilute limit, corresponding to $\hat{\Pi} \sim \varphi^{2}$ $N \sim\left(\varphi / \varphi_{\text {ov }}\right)^{2}$. So the two-dimensional equivalent of Equation (5) would be:

$$
\hat{\Pi}=1+\left(\varphi / \varphi_{\mathrm{ex}}\right)^{2}, \quad \varphi_{\mathrm{ex}}=c \varphi_{\mathrm{ov}}
$$

We test this equation against the simulation data of Dickman $^{[12]}$ for self-avoiding two-dimensional chains of length $N=40,80$, and 160. Dickman plots $\hat{\Pi}$ against $\varphi \sqrt{N}$, which equals $\varphi / \varphi_{\text {ov }}$ apart from a numerical constant; he does not report $R$ in the dilute limit nor the value of $\varphi_{\text {ov }}$. We use the data by Meirovitch, ${ }^{[32]}$ who gives $R=$

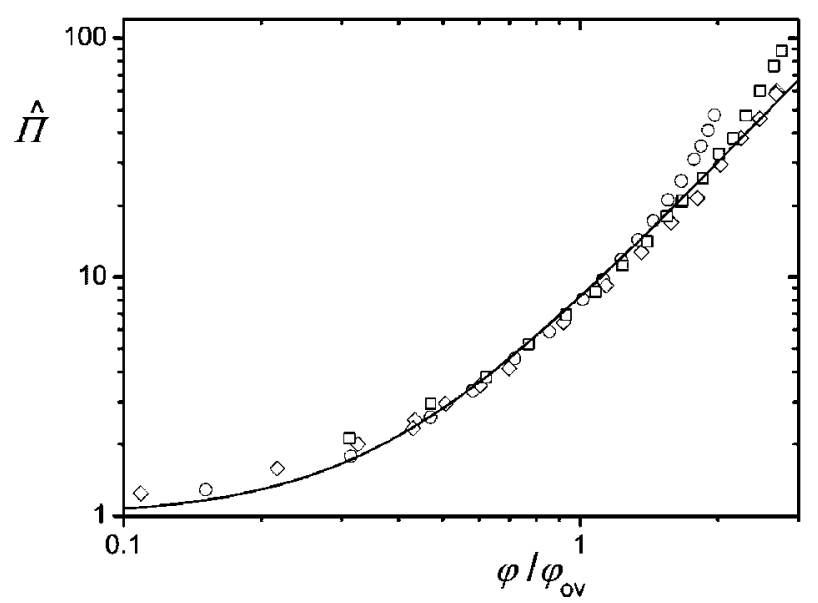

Figure 9. Comparison of the simulation data of Dickman ${ }^{[12]}$ for two-dimensional chains with Equation (30). The plot gives $\hat{\Pi}$ as a function of $\varphi / \varphi_{\text {ov }}$ on a double-logarithmic scale. Circles, squares, and diamonds are for $N=40,80$, and 160 , respectively. The solid curve is Equation (30) with $\varphi_{\mathrm{ex}}=0.37 \varphi_{\mathrm{ov}}$.

$0.339 N^{0.745}$. According to Equation (29a) this corresponds to $\varphi_{\text {ov }}=2.77 N^{-0.49}$. So we can make a plot $\hat{\Pi}\left(\varphi / \varphi_{\text {ov }}\right)$, which is given in Figure 9.

Extrapolating the semidilute line with slope 2 towards $\hat{\Pi}=1$ gives $\varphi_{\mathrm{ex}}=0.37 \varphi_{\mathrm{ov}}$. The constant $c$ is somewhat smaller than its three-dimensional equivalent $c=0.69$ (Equation 13), but our general extrapolation approach seems to work in two dimensions as well.

\section{Conclusion}

In this paper we suggest to use a linear combination of two limits (dilute and semidilute) to obtain an extremely simple equation that can describe the concentration dependence of the osmotic pressure and the depletion thickness over the entire range of concentrations. This approach also gives the transition from the dilute to the semidilute regime in a natural and uniform way. The only two parameters are the semidilute De Gennes exponent and the extrapolation concentration, both of which are easily accessible.

We demonstrate that there are two strategies to describe experimental data. When there are data in a concentration region far above the overlap concentration, they may be plotted as a function of the concentration in double-logarithmic form, and the limiting slope can be used as an input into our equation. It is also possible to extract this limiting slope from scaling theories. In both cases we can determine the intersection point of the two limits, which gives the extrapolation concentration and is the second parameter in our equation. Our 
simple equation allows manipulating it analytically, in contrast to the complicated equations from the grouprenormalization approach.

Our approach can be used for interpreting experimental results as well as data obtained by computer simulation in three (or two) dimensions. We expect that it can be applied in a compact presentation of the concentration dependence for several structural and thermodynamic properties, for example, the end-to-end distance, the radius of gyration, the correlation length, etc.

For some quantities, like the osmotic pressure $\Pi$, a linear combination of the quantity itself in the two limits is feasible, as we have shown in this paper. More generally, a combination rule for $X^{n}$, where $X$ is some property and $n$ is positive, gives the correct two limits when $X$ increases with concentration. The value of $n$ determines the behavior in the transition region; for $X=\Pi, n=1$ is adequate.

Other quantities, like the depletion thickness $\delta$, decrease with concentration. Then a combination rule for $X^{n}$ works only when $n$ is negative: we have to construct a linear combination of inverse quantities. In the case of the depletion thickness we need a combination of inverse squares $\delta^{-2}$, in line with earlier results. In a future paper we shall demonstrate that the same combination is natural for the concentration dependence of the correlation length.

Simple equations which describe the characteristics of a particular property can give a key to predictions for other properties of the same system. As an example, we demonstrated how from the equation for the osmotic pressure we can predict the osmotic compressibility and the structure factor $S(0)$.

Acknowledgements: This work was supported by the Dutch National Science Foundation (NWO) and the Russian Foundation for Basic Research (RFBR) through joint projects 047.016.004 and 047.017.026. A.M.S. acknowledges RFBR for financial support through project 05-03-32003a.

Received: March 2, 2007; Revised: March 26, 2007; Accepted: March 27, 2007; DOI: 10.1002/mats.200700022

Keywords: depletion thickness; osmotic pressure; polymer solutions; solution properties; thermodynamics

[1] P. J. Flory, "Principles of Polymer Chemistry", Cornell University Press, Ithaca, New York 1953.
[2] G. J. Fleer, M. A. Cohen Stuart, J. M. H. M. Scheutjens, T. Cosgrove, B. Vincent, "Polymers at Interfaces", Chapman \& Hall, London 1993.

[3] H. Yamakawa, "Modern Theory of Polymer Solutions", Harper and Row, New York 1971.

[4] G. J. Fleer, A. M. Skvortsov, R. Tuinier, Macromolecules 2003, 36, 7857.

[5] L. T. Lee, O. Guiselin, A. Lapp, B. Farnoux, Phys. Rev. Lett. 1991, 67, 2838.

[6] P.-G. De Gennes, "Scaling Concepts in Polymer Physics", Cornell University Press, Ithaca, New York 1979.

[7] J. Des Cloiseaux, G. Jannink, "Polymers in Solution", Oxford University Press, Oxford 1990.

[8] D. G. A. L. Aarts, R. Tuinier, H. N. W. Lekkerkerker, J. Phys.: Condens. Matter 2002, 14, 7551.

[9] W. W. Yau, J. J. Kirkland, D. D. Bly, "Modern Size Exclusion Liquid Chromatography", John Wiley, New York 1979.

[10] H. Pasch, B. Trathnigg, "HPLC of Polymers", Springer, Berlin 1997.

[11] A. Baumgärtner, "Application of the Monte Carlo Method in Statistical Polymers", K. Binder, Ed., Springer, New York 1984, Chap. 4.

[12] R. Dickman, J. Chem Phys. 1989, 91, 454; 1988, 89, 7577; 1987, 87, 2246.

[13] A. Milchev, W. Paul, K. Binder, J. Chem. Phys. 1993, 99, 4786.

[14] A. Pelisetto, E. Vicari, Phys. Rep. 2002, 368, 549.

[15] T. Ohta, Y. Oono, Phys. Lett. 1982, 89A, 460.

[16] A. Nakanishi, T. Ohta, J. Phys A: Math. Gen. 1985, 18, 127.

[17] L. Schäfer, Macromolecules 1984, 17, 1357.

[18] L. Schäfer, "Excluded Volume Effects in Polymer Solutions", Springer Verlag, Berlin 1999.

[19] I. Noda, N. Kato, T. Kitano, M. Nagasawa, Macromolecules 1981, 14, 688.

[20] P. G. Bolhuis, A. A. Louis, J.-P. Hansen, E. J. Meijer, J. Chem. Phys. 2001, 114, 4296

[21] A. A. Louis, P. G. Bolhuis, E. J. Meijer, J. Chem. Phys. 2002, 116, 10547.

[22] E. F. Casassa, J. Pol. Sci. B 1967, 5, 773; Macromolecules 1969, 2, 14.

[23] E. Eisenriegler, "Polymers near Surfaces", World Scientific, Singapore 1993.

[24] A. Hanke, E. Eisenriegler, S. Dietrich, Phys. Rev. E. 1999, 59, 6853.

[25] W. Y. Shih, W.-H. Shih, I. A. Aksay, Macromolecules 1990, 23, 3291.

[26] G. J. Fleer, A. M. Skvortsov, Macromolecules 2005, 38, 2492.

[27] P. Cifra, T. Bleha, J. Wang, I. Teraoka, J. Chem. Phys. 2000, 113, 8313.

[28] P. G. Bolhuis, A. A. Louis, Macromolecules 2002, 35, 1860.

[29] C. N. Satterfield, C. K. Colton, B. de Turckheim, T. M. Copeland, AIChE J. 1978, 24, 937.

[30] P. Wiltzius, H. R. Haller, D. S. Canell, Phys Rev. Lett. 1983, 51, 1183.

[31] F. Hamada, S. Kinugasa, H. Hayashi, A. Nakajima, Macromolecules 1985, 18, 2290.

[32] H. Meirovitch, Macromolecules. 1985, 18, 569. 